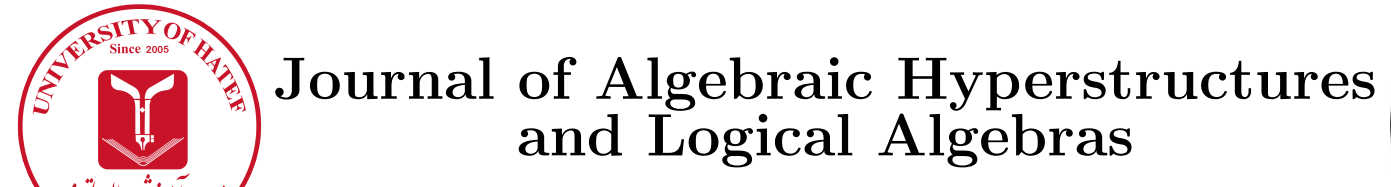

Special Issue (JAHA2020) Dedicated to Professor Piergiulio Corsini

Volume 1, Number 3, (2020), pp. 51-60

\title{
On strong-inverse elements
}

\author{
T. Kaplani ${ }^{1}$ and T. Vougiouklis ${ }^{2}$ \\ ${ }^{1}$ Fotada, 42100 Trikala, Greece, \\ ${ }^{2}$ Democritus University of Thrace, Neapoli 14-6, Xanthi 67100, Greece \\ dorakikaplani@gmail.com, tvougiou@eled.duth.gr
}

\begin{abstract}
Hyperstructures have applications in mathematics and in other sciences. For this, the largest class of the hyperstructures, the $H_{v}$-structures, is used. They satisfy the weak axioms where the non-empty intersection replaces equality. The fundamental relations connect, by quotients, the $H_{v}$-structures with the classical ones. Since the number of $H_{v}$-structures defined on the same set is very big, it is important to study special elements. A lot of those special elements are not appeared in the classical theory therefore, one has to discover their properties from the beginning. We continuous our study on $H_{v}$-structures which have the so called strong inverse elements.
\end{abstract}

Article Information

Corresponding Author:

T. Kaplani;

Received: July 2020;

Accepted: August 2020.

Paper type: Original.

\section{Keywords:}

Hyperstructure, hope, $H_{v^{-}}$ structure, strong inverse element.

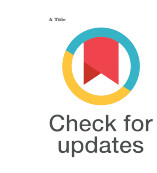

\section{Introduction}

In the hyperstructures it is not necessary to exist a unit element and if there exist, they can be more than one. Similarly, for a given element, the inverse element with respect to a unit element, there exist or not, and if there exist they can be more than one. We introduced in 2016 [14, the concept of strong inverse element which is defined to be an element if it is inverse, to a given element, with respect to all units. In any $H_{v}$-group, or any $h / v$-group, one can enlarge some results and the new hyperstructure remain $H_{v}$-group or $h / v$-group. In this paper we continue our study on $H_{v}$-groups with strong inverse elements by presenting constructions, mainly on finite sets, using enlargement, on special classes of $H_{v}$-groups. Notice that we remain on the same fundamental classes of the original hyperstructure.

https://doi.org/10.29252/HATEF.JAHLA.1.3.4 


\section{Preliminaries}

The hyperstructures called $H_{v}$-structures, introduced in 1990 [7], [8] by Vougiouklis, satisfy the weak axioms where the non-empty intersection replaces equality.

Definition 2.1. Algebraic hyperstructure $(H, \cdot)$ is a set $H$ with a hyperoperation (abbreviated hope) $\cdot: H \times H \longrightarrow P(H)-\{\emptyset\}$. We abbreviate by

WASS the weak associativity: $(x y) z \cap x(y z) \neq \emptyset, \forall x, y, z \in H$ and by

$C O W$ the weak commutativity: $x y \cap y x \neq \emptyset, \forall x, y \in H$.

The algebraic hyperstructure $(H, \cdot)$ is an $H_{v}$-semigroup if it is WASS, it is called $H_{v}$-group if it is reproductive $H_{v}$-semigroup, i.e. $x H=H x=H, \forall x \in H$.

The $(R,+, \cdot)$ is called $H_{v}$-ring if $(+)$ and $(\cdot)$ are WASS, the reproduction axiom is valid for $(+)$ and $(\cdot)$ is weak distributive to $(+)$ :

$$
x(y+z) \cap(x y+x z) \neq \emptyset \text { and }(x+y) z \cap(x z+y z) \neq \emptyset, \forall x, y, z \in R .
$$

For more definitions and applications on $H_{v^{-}}$structures one can see in books and papers as [1], [3], [4, 8], 11], [12], [13].

Definition 2.2. Let $(H, \cdot)$ and $(H, *)$ be two $H_{v}$-semigroups. Then the hope $(\cdot)$ is smaller than $(*)$, and $(*)$ is greater than $(\cdot)$, iff there exists an automorphism

$$
f \in \operatorname{Aut}(H, *) \text { such that } x y \subset f(x * y), \forall x, y \in H .
$$

We write $\cdot \leq *$ and say that $(H, *)$ contains $(H, \cdot)$. If $(H, \cdot)$ is a structure, then it is basic structure and $(H, *)$ is an $H_{b}$-structure.

The Little Theorem. Greater hopes than the ones which are WASS or COW, are WASS or COW, respectively.

The main tool in hyperstructures is the fundamental relation. M. Koskas in 1970 introduced in hypergroups the relation $\beta^{*}$, which connects hypergroups with groups and it is defined in $H_{v^{-}}$ groups as well. Vougiouklis [7], [8], [9], [11, introduced the $\gamma^{*}$ and $\epsilon^{*}$ relations, which are defined, in $H_{v}$-rings and $H_{v}$-vector spaces, respectively.

Definition 2.3. The fundamental relations $\beta^{*}, \gamma^{*}$ and $\epsilon^{*}$ are defined in $H_{v}$-groups, $H_{v}$-rings and $H_{v}$-vector spaces, respectively, as the smallest equivalences so that the quotient would be group, ring and vector spaces, respectively.

Theorem 2.4. Let $(H, \cdot)$ be an $H_{v}$-group and denote by $U$ the set of all finite products of elements of $H$. Define the relation $\beta$ in $H$ by: $x \beta y$ iff $\{x, y\} \subset u$ where $u \in U$. Then $\beta^{*}$ is the transitive closure of $\beta$.

Analogous theorems are for $H_{v}$-rings, $H_{v}$-vector spaces and so on.

An element is called single if its fundamental class is singleton [8].

Definition 2.5. An $H_{v}$-ring $(R,+, \cdot)$ is an $H_{v}$-field if $R / \gamma^{*}$ is a field.

Definition 2.6. [9] The $H_{v}$-semigroup $(H, \cdot)$ is called an $h / v$-group if $H / \beta^{*}$ is a group.

The $h / v$-group is not reproductive, so it is generalization of $H_{v}$-group. Similarly, $h / v$-rings, $h / v$-fields etc. are defined.

The uniting elements method, introduced by Corsini \& Vougiouklis in 1989, is the following [2], [8]: Let $G$ be a structure and not valid property $d$, described by a set of equations. Take the 
partition in $G$ for which put in the same class, all pairs of elements that causes the non-validity of $d$. The quotient by this partition $G / d$ is an $H_{v^{-}}$-structure. Then, quotient out $G / d$ by $\beta^{*}$, is a stricter structure $(G / d) / \beta^{*}$ for which $d$ is valid.

We remark that in $H_{v}$-groups, or even in hypergroups in the sense of $\mathrm{F}$. Marty, we do not have necessarily any 'unit' element, consequently neither 'inverses'. However, we may have, more than one, unit elements and for each element of an $H_{v}$-group we may have one inverse element or more than one.

Definition 2.7. Let $(H, \cdot)$ be an $H_{v}$-semigroup. An element $e$, it is called left unit if $x \in e x, \forall x \in$ $H$, it is called right unit if $x \in x e, \forall x \in H$ and it is called unit element if it is both left and right unit element. For given unit $e$, an element $x \in H$, has a left inverse with respect to $e$, any element $x_{l e}$ if $e \in x_{l e} \cdot x$, it has a right inverse element $x_{r e}$ if $e \in x \cdot x_{r e}$, and it has an inverse $x_{e}$ with respect to $e$, if $e \in\left(x_{e} \cdot x\right) \cap\left(x \cdot x_{e}\right)$. Denote by $E_{l}$ the set of all left unit elements, by $E_{r}$ the set of all right unit elements, and by $E$ the set of unit elements.

Definition 2.8. [5, 14] Let $(H, \cdot)$ be an $H_{v}$-semigroup. An element is called strong-inverse if it is an inverse to $x$ with respect to all unit elements. Thus, an element $x_{s}$ it is a strong-inverse to $x$, if

$$
E \subset\left(x_{s} \cdot x\right) \cap\left(x \cdot x_{s}\right)
$$

\section{Some classes of hopes}

A class of $H_{v}$-structures, introduced in [8], is the following:

Definition 3.1. An $H_{v}$-structure is called very thin if all hopes are operations except one, which has all hyperproducts singletons except one, which is a subset of cardinality more than one. Thus, in a very thin $H_{v}$-structure in $H$ there exists a hope $(\cdot)$ and a pair $(a, b) \in H^{2}$ for which $a b=A$, with $\operatorname{card} A>1$, and all the other products, are singletons.

Another large class is the one on which a new hope $(\partial)$ in a groupoid is defined.

Definition 3.2. [10, 11, 4]. Let $(G, \cdot)$ be groupoid (resp., hypergroupoid) and $f: G \longrightarrow G$ be a map. We define a hope $(\partial)$, called theta-hope or simply $\partial$-hope, on $G$ as follows,

$$
x \partial y=\{f(x) \cdot y, x \cdot f(y)\}, \forall x, y \in G .(\text { resp. } x \partial y=(f(x) \cdot y) \cup(x \cdot f(y)), \forall x, y \in G .)
$$

If $(\cdot)$ is commutative, then $(\partial)$ is commutative. If $(\cdot)$ is $C O W$, then $(\partial)$ is $C O W$.

Let $(G, \cdot)$ be a groupoid (resp. hypergroupoid) and $f: G \longrightarrow P(G)-\{\emptyset\}$ be a multivalued map. We define the hope $(\partial)$ on $G$ as follows,

$$
x \partial y=(f(x) \cdot y) \cup(x \cdot f(y)), \forall x, y \in G .
$$

Properties. If $(G, \cdot)$ is a semigroup, then for any $f$, the hope $(\partial)$ is WASS. If $f$ is homomorphism and projection, or idempotent: $f^{2}=f$, then $(\partial)$ is associative.

Let $(G, \cdot)$ be a groupoid and $f_{i}: G \longrightarrow G, i \in I$, be a set of maps on $G$. We consider the map $f_{\cup}: G \longrightarrow P(G)$ such that $f \cup(x)=\left\{f_{i}(x) \mid i \in I\right\}$, called the union of the $f_{i}(x)$. We define the union $\partial$-hope, on $G$ if we consider as map the $f \cup(x)$. A special case is if we consider the map $\underline{f} \equiv f \cup(i d)$, so $\underline{f}(x)=\{x, f(x)\}, \forall x \in G$, which we call $b$ - $\partial$-hope. Then we have

$$
x \partial y=\{x y, f(x) \cdot y, x \cdot f(y)\}, \forall x, y \in G .
$$


Proposition 3.3. Let $(G, \cdot)$ be a group and for any $x \in G, f(x)=a$. Then $(G, \partial) / \beta^{*}$ is a singleton.

Proof. For all $x \in G$, we can take the hyperproduct of the elements, $a^{-1}$ and $a^{-1} x$

$$
a^{-1} \partial\left(a^{-1} \cdot x\right)=\left\{f\left(a^{-1}\right) \cdot a^{-1} \cdot x, a^{-1} \cdot f\left(a^{-1} \cdot x\right)\right\}=\{x, a\} .
$$

Thus $x \beta a, \forall x \in G$, so $\beta^{*}(x)=\beta^{*}(a)$ and $(G, \partial) / \beta^{*}$ is singleton.

Special case if $(G, \cdot)$ is a group and $f(x)=e$, then $x \theta y=\{x, y\}$.

The application on the derivative: On the polynomials $g_{i}(x)=a_{i} x+b_{i}$, take the hope

$$
g_{1} \partial g_{2}=\left\{a_{1} a_{2} x+a_{1} b_{2}, a_{1} a_{2} x+b_{1} a_{2}\right\} .
$$

All polynomials $x+c$, where $c$ a constant, are units.

Example 3.4. (a) In integers $(Z,+, \cdot)$ fix $n \neq 0$, a natural number. Consider the map $f$ such that $f(0)=n$ and $f(x)=x, \forall x \in Z-\{0\}$. Then $\left(Z, \partial_{+}, \partial \cdot\right)$, where $\partial_{+}$and $\partial$. are the $\partial$-hopes refereed to the addition and the multiplication respectively, is an $H_{v}$-near-ring, with

$$
\left(Z, \partial_{+}, \partial \cdot\right) / \gamma^{*} \cong Z_{n}
$$

(b) In $(Z,+, \cdot)$ with $n \neq 0$, take $f$ such that $f(n)=0$ and $f(x)=x, \forall x \in Z-\{n\}$. Then $\left(Z, \partial_{+}, \partial \cdot\right)$ is an $H_{v}$-ring, moreover, $\left(Z, \partial_{+}, \partial \cdot\right) / \gamma^{*} \cong Z_{n}$.

Special case of the above is for $n=p$, prime, then $\left(Z, \partial_{+}, \partial \cdot\right)$ is an $H_{v}$-field.

Theorem 3.5. In the ring $\left(Z_{n},+, \cdot\right)$, with $n=m s$ enlarge the multiplication only in the product of the elements $0 \cdot m$ by setting $0 \otimes m=\{0, m\}$ and the rest results remain the same. Then

$$
\left(Z_{n},+, \otimes\right) / \gamma^{*} \cong\left(Z_{m},+, \cdot\right) \text {. }
$$

Remark that we can enlarge other products as well, for example $2 \cdot m$ by setting $2 \cdot m=\{2, m+2\}$, then the result remains the same. In this case 0 and 1 are scalars.

Corollary 3.6. In the ring $(Z n,+, \cdot)$, with $n=p s$, where $p$ is prime, we enlarge only the product $0 \cdot p$ by $0 \otimes p=\{0, p\}$ and the rest remain the same. Then $\left(Z_{n},+, \otimes\right)$ is very thin $H_{v}$-field.

Proof. The only one hyperproduct $0 \otimes p=\{0, p\}$ in $\left(Z_{n},+, \otimes\right)$ give the following $p$ fundamental classes

$$
\{0, p, 2 p, \ldots,(s-1) p\},\{1, p+1,2 p+1, \ldots,(s-1) p+1\}, \ldots,\{p-1,2 p-1,3 p-1, \ldots, s p-1\} .
$$

Therefore, $\left(Z_{n},+, \otimes\right)$ is an $H_{v}$-field, with fundamental field the $\left(Z_{p},+, \cdot\right)$.

The large class of $P$-hyperstructures was appeared in 80's to represent hopes of constant length [4], 6], 8], [14].

Definition 3.7. Let $(G, \cdot)$ be a groupoid. Then for all $P$ such that $\emptyset \neq P \subset G$, we define the following hopes called P-hopes: $\forall x, y \in G$

$$
\begin{gathered}
\underline{P}: x \underline{P} y=(x P) y \cup x(P y), \\
\underline{P}_{r}: x \underline{P}_{r} y=(x y) P \cup x(y P), \\
\underline{P}_{l}: x \underline{P}_{l} y=(P x) y \cup P(x y) .
\end{gathered}
$$

The $(G, \underline{P}),\left(G, \underline{P}_{r}\right)$ and $\left(G, \underline{P}_{l}\right)$ are called P-hyperstructures. The most usual case is when $(G, \cdot)$ is semigroup, then we have 


$$
x \underline{P} y=(x P) y \cup x(P y)=x P y,
$$

and $(G, \underline{P})$ is a semihypergroup.

It is immediate the following: Let $(G, \cdot)$ be a group, then for all subsets $P$ such that $\emptyset \neq P \subset G$, the hyperstructure $(G, \underline{P})$, where the $P$-hope is $x \underline{P} y=x P y$, becomes a hypergoup in the sense of Marty, i.e. the strong associativity is valid. The $P$-hope is of constant length, i.e. we have $|x \underline{P} y|=|P|$. We call the hyperstructure $(G, \underline{P})$, P-hypergroup.

A modified $P$-hope was introduced which is appropriate for the $e$-hyperstructures:

Construction 3.8. Let $(G, \cdot)$ be an Abelian group and $P$ any subset of $G$ with more than one elements. We define the hyperoperation $\times_{P}$ as follows:

$$
x \times_{P} y=\left\{\begin{array}{ll}
x \cdot P \cdot y=\{x \cdot h \cdot y \mid h \in P\} & \text { if } x \neq e \text { and } c \neq e \\
x \cdot y & \text { if } x=e \text { or } y=e
\end{array} .\right.
$$

We call this hope $P_{e}$-hope. The hyperstructure $\left(G, \times_{P}\right)$ is an Abelian $H_{v}$-group.

\section{Strong-inverse}

We present some results and examples of hyperstructures with strong-inverse elements.

Properties 4.1. Let $(G, \cdot)$ be a group, take $P$ such that $\emptyset \neq P \subset G$ and the $P$-hypergroup $(G, \underline{P})$, where $x \underline{P} y=x P y$. We have the following:

Units: In order an element $u$ to be right unit of the $P$-hypergroup $(G, \underline{P})$, we must have $x \underline{P} u=x P u \ni x, \forall x \in G$. In fact, the set $P u$ must contain the unit element $e$ of the group $(G, \cdot)$. Thus, all the elements of the set $P^{-1}$, are right units. The same is valid for the left units, therefore, the set of all units is the $P^{-1}$.

Inverses: Let $u$ be a unit in $(G, \underline{P})$, then, for given $x$ in order to have an inverse element $x$ with respect to $u$, we must have $x \underline{P} x^{\prime}=x P x^{\prime} \ni u$, so taking $x p x^{\prime}=u$, we obtain that all the elements of the form $x^{\prime}=p^{-1} x^{-1} u$ are inverses to $x$ with respect to the unit $u$.

Theorem 4.2. 14] Let $(G, \cdot)$ be a group, then for all normal subgroups $P$ of $G$, the hyperstructure $(G, \underline{P})$, where $x \underline{P} y=x P y, \forall x, y \in G$, is a hypergoup with strong-inverses. Moreover, for any inverse $x^{\prime}$ of $x \in G$, with respect to any unit, we have $x \underline{P} x^{\prime}=P$.

Proof. Let $x \in G$. Take an inverse $x^{\prime}=p^{-1} x^{-1} u$ with respect to the unit $u=p_{k}^{-1}$, for any $p$. Then we have $x \underline{P} x^{\prime}=x P x^{\prime}$. But, since $P$ is normal subgroup, we have

$$
x \underline{P} x^{\prime}=x p^{-1} x^{-1} p_{k}^{-1} P=x p^{-1} x^{-1} P=x p^{-1} P x^{-1}=x P x^{-1}=P .
$$

Remark that in this case, $P^{-1}=P$, is the set of all units, thus all inverses are strong.

Proposition 4.3. Let $(G, \cdot)$ be a groupoid, $f: G \longrightarrow G$ be a map and $(G, \partial)$ the corresponding $\partial$-structure, then we have the following:

Units: In order an element $u$ to be right unit, we must have

$$
x \partial u=\{f(x) \cdot u, x \cdot f(u)\} \ni x .
$$

The unit not depend on $f(x)$, so $f(u)=e$, where $e$ be unit in the monoid $(G, \cdot)$. The same it is obtained for the left units. So the elements of kern $f=\{u: f(u)=e\}$, are the units of $(G, \partial)$.

Inverses: Let $u$ be a unit in $(G, \partial)$, then $(G, \cdot)$ is a monoid with unit e and $f(u)=e$. For given $x$ in order to have an inverse element $x^{\prime}$ with respect to $u$, we must have 


$$
x \partial x^{\prime}=\left\{f(x) \cdot x^{\prime}, x \cdot f\left(x^{\prime}\right)\right\} \ni u \text { and } x^{\prime} \partial x=\left\{f\left(x^{\prime}\right) \cdot x, x^{\prime} \cdot f(x)\right\} \ni u .
$$

So, the only cases, which do not depend on the image $f\left(x^{\prime}\right)$, are

$$
x^{\prime}=(f(x))^{-1} u \text { and } x^{\prime}=u(f(x))^{-1}
$$

the right and left inverses, respectively. We have two-sided inverses iff $f(x) u=u f(x)$.

Remark [13]: Since the inverses are depending on the units, thus they are not strong.

The following constructions [14], are obtained from properties the strong-inverse elements have. This is an enlargement in order all the elements to be strong-inverses.

Construction 4.4. Let $(G, \cdot)$ be a group with unit $e$. Consider a finite set $E=\left\{e_{i} \mid i \in I\right\}$. On the set $\underline{G}=(G-\{e\}) \cup E$ we define a hope $(\times)$ as follows:

$$
\left\{\begin{array}{l}
e_{i} \times e_{j}=\left\{e_{i}, e_{j}\right\}, \forall e_{i}, e_{j} \in E \\
e_{i} \times x=x \times e_{i}=x, \forall e_{i} \in E, \forall x \in G-\{e\} \\
x \times y=x \cdot y \text { if } x \cdot y \in G-\{e\} \text { and } x \times y=E \text { if } x \cdot y=e .
\end{array}\right.
$$

Then the hyperstructure $(\underline{G}, \times)$ is a hypergroup. The set of unit elements is $E$ and all the elements are strong-inverse. Moreover we have $(\underline{G}, \times) / \beta^{*} \cong(G, \cdot)$.

Proof. For the associativity we have the cases:

$$
\begin{gathered}
\left(e_{i} \times e_{j}\right) \times e_{k}=e_{i} \times\left(e_{j} \times e_{k}\right)=\left\{e_{i}, e_{j}, e_{k}\right\}, \forall e_{i}, e_{j}, e_{k} \in E \\
(x \times y) \times z=x \times(y \times z)=x \cdot y \cdot z \text { or } E, \forall x, y, z \in \underline{G} \text { and not all of them belong to } E .
\end{gathered}
$$

In the second case the product of two inverses can appear. The only difference is that the result is singleton and in some cases the result is the set $E$. Thus, the strong associativity is valid. The reproductivity is valid and $E$ is the set of units in $(\underline{G}, \times)$.

Two elements of $\underline{G}$ are $\beta^{*}$ equivalent if they belong to any finite $\times$-product of elements of $\underline{G}$. Therefore, all fundamental classes are singletons except the set of units $E$. That means that we have $(\underline{G}, \times) / \beta^{*} \cong(G, \cdot)$.

Construction 4.5. Let $(G, \cdot)$ be an $H_{v}$-group with one unit $e$ and every element has unique inverse. Consider a finite set $E=\left\{e_{i} \mid i \in I\right\}$. On the set $\underline{G}=(G-\{e\}) \cup E$ we define a hope $(\times)$ as follows:

$$
\left\{\begin{array}{l}
e_{i} \times e_{j}=\left\{e_{i}, e_{j}\right\}, \forall e_{i}, e_{j} \in E \\
e_{i} \times x=x \times e_{i}=x, \forall e_{i} \in E, \forall x \in G-\{e\} \\
x \times y=x \cdot y \text { if } x \cdot y \in G-\{e\} \text { and } x \times y=E \text { if } x \cdot y=e
\end{array}\right.
$$

Then the hyperstructure $(\underline{G}, \times)$ is an $H_{v}$-group. The set of unit elements is $E$ and all the elements are strong-inverse. Moreover, we have

$$
(\underline{G}, \times) / \beta^{*} \cong(G, \cdot) / \beta * .
$$

Proof. For the associativity we have the cases:

$$
\begin{gathered}
\left(e_{i} \times e_{j}\right) \times e_{k}=e_{i} \times\left(e_{j} \times e_{k}\right)=\left\{e_{i}, e_{j}, e_{k}\right\}, \forall e_{i}, e_{j}, e_{k} \in E . \\
(x \times y) \times z=x \times(y \times z)=x \cdot y \cdot z \text { or } E, \forall x, y, z \in \underline{G} \text { and not all of them belong to } E .
\end{gathered}
$$


Therefore, the WASS is valid. Moreover, the reproductivity is valid and the set $E$ is the set of units in $(\underline{G}, \times)$.

Two elements of $\underline{G}$ are $\beta^{*}$ equivalent if they belong to any finite $\times$-product of elements of $\underline{G}$. So, all fundamental classes correspond to the fundamental classes of $(G, \cdot)$, with an enlargement of the class of $e$ into $E$. Thus, we have $(\underline{G}, \times) / \beta^{*} \cong(G, \cdot) / \beta^{*}$.

We remark that the above constructions give a great number of hyperstructures with stronginverses because we can enlarge then in any result except if the result is $E$.

Now, we present a result on strong-inverses on a general finite case, see [5].

Theorem 4.6. A minimum non-degenerate, i.e. have non-degenerate fundamental field, $h / v$-field with strong-inverses with respect to sum-hope, obtained by enlarging the ring $\left(Z_{p q},+, \cdot\right)$, where $p, q$ is prime numbers, and which has fundamental field isomorphic to $\left(Z_{q},+, \cdot\right)$, is defined as follows:

The sum-hope $(\oplus)$ is enlarged from $(+)$ by setting

1. $q \oplus x=x \oplus q=\{x, x+q\}, \forall x \in Z_{p q}$,

2. whenever, except for $0 \oplus 0=0$, the result is 0 and $q$ we enlarge it by setting $q$ and 0 , respectively.

Then, the set of zeros is $E=\{0, q\}$, the fundamental classes are

$$
\underline{x}=\{x, x+q, x+2 q, \ldots, x+(p-1) q\}, \forall k \in Z_{p q}
$$

and

$$
\left(Z_{p q}, \oplus, \cdot\right) / \gamma^{*} \cong\left(Z_{q},+, \cdot\right)
$$

Proof. We have $p q$ elements and we want to have the zero-set $E=\{0, q\}$. In order to have strongopposite, according to Definition 1.6, whenever the result is 0 and $q$ we enlarge it by setting $q$ and 0 , respectively. Thus, we have

$$
E \subset\left(x_{s} \oplus x\right) \cap\left(x \oplus x_{s}\right), \forall x \in Z_{p q} \text {, where } x_{s} \text { is the strong-opposite of } x .
$$

From the above definition of the sum-hope it is easy to prove that the fundamental classes are of the form

$$
\underline{x}=\{x, x+q, x+2 q, \ldots, x+(p-1) q\}, \forall x \in Z_{p q} .
$$

Therefore, it is obtained that $\left(Z_{p q}, \oplus, \cdot\right)$ it is an $h / v$-field and its fundamental field it is isomorphic to $\left(Z_{q},+, \cdot\right)$.

Remark 4.7. We remark that if we take, for example, the triple $1,2, q-2$, we have

$$
\begin{aligned}
& (1 \oplus 2) \oplus(q-2)=3 \oplus(q-2)=q+1 \text { and } \\
& 1 \oplus(2 \oplus(q-2))=1 \oplus\{q, 0\}=\{q+1,1\} .
\end{aligned}
$$

Therefore, $\oplus$ it is WASS, not strong associative.

As example of the above theorem we present the following case:

Example 4.8. In the case of the $h / v$-field $\left(Z_{15}, \oplus, \cdot\right)$, where $p=3, q=5$, we have the table: and moreover, it is easy to see that the fundamental field is isomorphic to $\left(Z_{5},+, \cdot\right)$. 


\begin{tabular}{|l|l|l|l|l|l|l|l|l|l|l|l|l|l|l|l|}
\hline & $\mathbf{0}$ & $\mathbf{1}$ & $\mathbf{2}$ & $\mathbf{3}$ & $\mathbf{4}$ & $\mathbf{5}$ & $\mathbf{6}$ & $\mathbf{7}$ & $\mathbf{8}$ & $\mathbf{9}$ & $\mathbf{1 0}$ & $\mathbf{1 1}$ & $\mathbf{1 2}$ & $\mathbf{1 3}$ & $\mathbf{1 4}$ \\
\hline $\mathbf{0}$ & 0 & 1 & 2 & 3 & 4 & 5,0 & 6 & 7 & 8 & 9 & 10 & 11 & 12 & 13 & 14 \\
\hline $\mathbf{1}$ & 1 & 2 & 3 & 4 & 5,0 & 6.1 & 7 & 8 & 9 & 10 & 11 & 12 & 13 & 14 & 0,5 \\
\hline $\mathbf{2}$ & 2 & 3 & 4 & 5,0 & 6 & 7.2 & 8 & 9 & 10 & 11 & 12 & 13 & 14 & 0,5 & 1 \\
\hline $\mathbf{3}$ & 3 & 4 & 5,0 & 6 & 7 & 8,3 & 9 & 10 & 11 & 12 & 13 & 14 & 0,5 & 1 & 2 \\
\hline $\mathbf{4}$ & 4 & 5,0 & 6 & 7 & 8 & 9,4 & 10 & 11 & 12 & 13 & 14 & 0,5 & 1 & 2 & 3 \\
\hline $\mathbf{5}$ & 5,0 & 6,1 & 7,2 & 8,3 & 9,4 & 10,5 & 11,6 & 12,7 & 13,8 & 14,9 & 0,10 & 1,11 & 2,12 & 3,13 & 4,14 \\
\hline $\mathbf{6}$ & 6 & 7 & 8 & 9 & 10 & 11,6 & 12 & 13 & 14 & 0,5 & 1 & 2 & 3 & 4 & 5,0 \\
\hline $\mathbf{7}$ & 7 & 8 & 9 & 10 & 11 & 12,7 & 13 & 14 & 0,5 & 1 & 2 & 3 & 4 & 5,0 & 6 \\
\hline $\mathbf{8}$ & 8 & 9 & 10 & 11 & 12 & 13,8 & 14 & 0,5 & 1 & 2 & 3 & 4 & 5,0 & 6 & 7 \\
\hline $\mathbf{9}$ & 9 & 10 & 11 & 12 & 13 & 14,9 & 0,5 & 1 & 2 & 3 & 4 & 5,0 & 6 & 7 & 8 \\
\hline $\mathbf{1 0}$ & 10 & 11 & 12 & 13 & 14 & 0,10 & 1 & 2 & 3 & 4 & 5,0 & 6 & 7 & 8 & 9 \\
\hline $\mathbf{1 1}$ & 11 & 12 & 13 & 14 & 0,5 & 1,11 & 2 & 3 & 4 & 5,0 & 6 & 7 & 8 & 9 & 10 \\
\hline $\mathbf{1 2}$ & 12 & 13 & 14 & 0,5 & 1 & 2,12 & 3 & 4 & 5,0 & 6 & 7 & 8 & 9 & 10 & 11 \\
\hline $\mathbf{1 3}$ & 13 & 14 & 0,5 & 1 & 2 & 3,13 & 4 & 5,0 & 6 & 7 & 8 & 9 & 10 & 11 & 12 \\
\hline $\mathbf{1 4}$ & 14 & 0,5 & 1 & 2 & 3 & 4,14 & 5,0 & 6 & 7 & 8 & 9 & 10 & 11 & 12 & 13 \\
\hline
\end{tabular}

Remark 4.9. From the fact that $\left(Z_{p q},+\right)$ is cyclic obtain that the $H_{v}$-group $\left(Z_{p q}, \cdot\right)$ is cyclic as well. We obtain that generators are the elements 1, 2, 4, 7, 8, 11, 13 and 14 and all of them are of period 14. Notice that the elements 0,5 and 10, belong to the zero class, therefore the elements 3, 6, 9 and 12 are not generators.

Theorem 4.10. A minimum non-degenerate $h / v$-field with strong-inverses with respect to producthope, obtained by enlarging the ring $\left(Z_{p q},+, \cdot\right)$, where $p, q$ is prime numbers, and which has fundamental field isomorphic to $\left(Z_{q},+, \cdot\right)$, is defined as follows:

The product-hope $(\otimes)$ is enlarged from $(+)$ by setting,

1. $(q+1) \otimes x=x \otimes(q+1) \ni\{x, x+x q\}, \forall x Z_{p q}$,

2. whenever, except for $1 \otimes 1=1$, the result is 1 and $q+1$ we enlarge it by setting $q+1$ and 1, respectively.

Then, the set of units is $U=\{1, q+1\}$, the fundamental classes are

$$
\underline{x}=\{x, x+q, x+2 q, \ldots, x+(p-1) q\}, \forall x \in Z_{p q}
$$

and

$$
\left(Z_{p q},+, \otimes\right) / \gamma^{*} \cong\left(Z_{q},+, \cdot\right) .
$$

Proof. We have $p q$ elements and we want to have the unit-set $U=\{1, q+1\}$. In order to have strong-inverse elements, according to Definition 1.5, whenever the result is 1 or $q+1$, we enlarge it by setting $q+1$ or 1 , respectively. From this definition of the product-hope it is easy to prove, by using the addition on the enlarged results, that the fundamental classes are of the form

$$
\underline{x}=\{x, x+q, x+2 q, \ldots, x+(p-1) q\}, \forall x Z_{p q} .
$$

On the product-hope of the sets of two classes $\underline{x}$ and $\underline{y}$, since their elements are $\bmod q$ equivalent, it is clear that we obtain that $x \otimes y \subset x y$.

Moreover, we have

$$
U \subset\left(x_{s} \otimes x\right) \cap\left(x \otimes x_{s}\right), \forall x \in Z_{p q}, \text { where } x_{s} \text { is the strong-inverse of } x .
$$


Therefore, $\left(Z_{p q},+, \otimes\right)$ is an $h / v$-field and $\left(Z_{p q},+, \otimes\right) / \gamma^{*} \cong\left(Z_{q},+, \cdot\right)$. This $h / v$-field in the product-hope has strong inverses.

Remark 4.11. In the above theorem, if we exclude the zero class $\underline{x}=\{0, q, 2 q, \ldots,(p-1) q\}$, we can write the multiplicative table to see that there is a reproductivity on classes and several other properties that there are on $h / v$-groups and there are no in the $H_{v}$-groups.

Remark 4.12. Let us denote $q^{\prime}=(q+1) / 2$, then taking the elements $2, q^{\prime}, q-1$, we have

$$
\begin{gathered}
\left(2 \otimes q^{\prime}\right) \otimes(q-1)=\{1, q+1\} \otimes(q-1)=\{q-1, q q-1\} \text { and } \\
2 \otimes\left(q^{\prime} \otimes(q-1)\right)=2 \otimes((q q-1) / 2)=q q-1
\end{gathered}
$$

Therefore, it is WASS, not strong associative.

Example 4.13. In the case of the $h / v$-field $\left(Z_{15},+, \otimes\right)$, where $p=3, q=5$, we have the following table on the set $Z_{15}-\{0,5,10\}$ :

\begin{tabular}{|l|l|l|l|l|l|l|l|l|l|l|l|l|}
\hline & $\mathbf{1}$ & $\mathbf{2}$ & $\mathbf{3}$ & $\mathbf{4}$ & $\mathbf{6}$ & $\mathbf{7}$ & $\mathbf{8}$ & $\mathbf{9}$ & $\mathbf{1 1}$ & $\mathbf{1 2}$ & $\mathbf{1 3}$ & $\mathbf{1 4}$ \\
\hline $\mathbf{1}$ & 1 & 2 & 3 & 4 & 6,1 & 7 & 8 & 9 & 11 & 12 & 13 & 14 \\
\hline $\mathbf{2}$ & 2 & 4 & 6,1 & 8 & 12,2 & 14 & 1,6 & 3 & 7 & 9 & 11 & 13 \\
\hline $\mathbf{3}$ & 3 & 6,1 & 9 & 12 & 3 & 6,1 & 9 & 12 & 3 & 6,1 & 9 & 12 \\
\hline $\mathbf{4}$ & 4 & 8 & 12 & 1,6 & 9,4 & 13 & 2 & 6,1 & 14 & 3 & 7 & 11 \\
\hline $\mathbf{6}$ & 6,1 & 12,2 & 3 & 9,4 & 6,1 & 12,7 & 3,8 & 9 & $6,11,1$ & 12 & 3,13 & 9,14 \\
\hline $\mathbf{7}$ & 7 & 14 & 6,1 & 13 & 12,7 & 4 & 11 & 3 & 2 & 9 & 1,6 & 8 \\
\hline $\mathbf{8}$ & 8 & 1,6 & 9 & 2 & 3,8 & 11 & 4 & 12 & 13 & 6,1 & 14 & 7 \\
\hline $\mathbf{9}$ & 9 & 3 & 12 & 6,1 & 9 & 3 & 12 & 6,1 & 9 & 3 & 12 & 6,1 \\
\hline $\mathbf{1 1}$ & 11 & 7 & 3 & 14 & $6,11,1$ & 2 & 13 & 9 & 1,6 & 12 & 8 & 4 \\
\hline $\mathbf{1 2}$ & 12 & 9 & 6,1 & 3 & 12 & 9 & 6,1 & 3 & 12 & 9 & 6,1 & 3 \\
\hline $\mathbf{1 3}$ & 13 & 11 & 9 & 7 & 3,13 & 1,6 & 14 & 12 & 8 & 6,1 & 4 & 2 \\
\hline $\mathbf{1 4}$ & 14 & 13 & 12 & 11 & 9,14 & 8 & 7 & 6,1 & 4 & 3 & 2 & 1,6 \\
\hline
\end{tabular}

Remark 4.14. We can see that $h / v$-group is not cyclic. However, if we consider the fundamental classes instead of elements, we can consider the powers of these sets, by multiplying all the elements of the set of a fundamental class, as in the following example,

$$
\begin{aligned}
& \{2,7,12\}^{1}=\{2,7,12\}=[2] \\
& \{2,7,12\}^{2}=\{4,14,9\}=[4] \\
& \{2,7,12\}^{3}=\{8,13,3\}=[3] \\
& \{2,7,12\}^{4}=\{1,11,6\}=[1] .
\end{aligned}
$$

Therefore, we obtain that in this sense, the set $[2]=\{2,7,12\}$ is a generator of period 4 . 


\section{Conclusions}

On the classes of the $H_{v}$-groups and $h / v$-groups we deal with the strong inverse elements which are elements which are inverse, to given element, with respect to all units. We presented constructions, mainly on finite sets, using enlargement, on special classes of $H_{v}$-groups and $h / v$-groups. These hyperstructures contain strong inverse elements and remain on the same fundamental classes of the original hyperstructure.

\section{References}

[1] P. Corsini, V. Leoreanu, Application of hyperstructure theory, Kluwer Academic Publishers, 2003.

[2] P. Corsini, T. Vougiouklis, From groupoids to groups through hypergroups, Rendiconti Mathemathics, S. VII, 9 (1989), 173-181.

[3] B. Davvaz, V. Leoreanu, Hyperring theory and applications, International Academic Press, 2007.

[4] B. Davvaz, T. Vougiouklis, A walk through weak hyperstructures, $H_{v}$-structures, World Scientific, 2018.

[5] T. Kaplani, T. Vougiouklis, On the finite $H_{v}$-fields with strong-inverses, Ratio Mathematica, 33 (2017), 115-126.

[6] T. Vougiouklis, Generalization of P-hypergroups, Rendiconti del Circolo Matematico di Palermo Series 2, 36 (1987), 114-121.

[7] T. Vougiouklis, The fundamental relation in hyperrings. The general hyperfield, $4^{\text {th }}$ AHA, Xanthi 1990, World Scientific, (1991), 203-211.

[8] T. Vougiouklis, Hyperstructures and their representations, Monographs in Mathematics, Hadronic, 1994.

[9] T. Vougiouklis, The $h / v$-structures, Journal of Discrete Mathematics, Sciences and Cryptography, 6(2-3) (2003), 235-243.

[10] T. Vougiouklis, Operations and $H_{v}$-fields, Acta Mathematica Sinica, (Engl. Ser.), 24(7) (2008), 1067-1078.

[11] T. Vougiouklis, On the hyperstructure theory, Southeast Asian Bulletin of Mathematics, 40(4) (2016), 603-620.

[12] T. Vougiouklis, $H_{v}$-fields, $h / v$-fields, Ratio Mathematica, 33 (2017), 181-201.

[13] T. Vougiouklis, Fundamental relations in $H_{v}$-structures. The Judging from the results' proof, Journal of Algebraic Hyperstrucures and Logical Algebras (JAHLA), 1(1) (2020), 21-36.

[14] T. Vougiouklis, T. Kaplani, Special elements on P-hopes and $\vartheta$-hopes, Southeast Asian Bulletin Mathematics, 40(3) (2016), 451-460. 